\title{
Expression of Death Receptors and Associated Regulatory Proteins in Pediatric Acute Lymphoblastic Leukemia: Preliminary Results
}

\author{
Bruce Musgrave*, Dorothy Barnard, David Hoskin \\ *Dalhousie Medical School Class of 2005
}

\begin{abstract}
Altered regulation of the cell cycle and/or apoptosis are well established events in the process of neoplastic transformation. Fas-mediated apoptosis is regulated by several inhibitory proteins in normal lymphocytes and leukemic cells. Some of these protein molecules may also play a role in the induction of apoptosis through death receptors for a similar molecule, TNF-related apoptosis inducing ligand (TRAlL). The involvement of these anti-apoptotic molecules in leukemogenesis is uncertain. Our preliminary findings indicate decreased expression of pro-apoptotic TRAIL-DR4 receptors in leukemic T cells relative to untransformed T cells. This decrease in expression may represent a potential determinant of poor acute lymphoblastic leukemia (ALL) prognosis, as it may result in a favorable biological situation for escape from apoptosis. Our data also demonstrate decreased levels of expression of pro-apoptotic Fas protein molecules in pediatric patient samples, relative to ALL cell lines. This difference suggests that circulating leukemic cells differ in their susceptibility to Fas-mediated apoptosis. Taken together, our data suggest that ALL cell lines and circulating leukemic cells from pediatric patients possess different regulatory mechanisms in order to attenuate Fas-mediated signaling.
\end{abstract}

$\mathrm{T}$ Traditionally, prognostic data for pediatric leukemia patients is obtained from routine physical examination, bone marrow morphology, and peripheral blood counts of pediatric leukemia patients. With recent developments in the understanding of molecular and cellular events in leukemia, information obtained via karyotyping, molecular genetics, and surface immunophenotype has also been useful for determining the optimal course of treatment along with the likelihood of treatment success. ${ }^{1-3}$ By determining the molecular composition of various leukemic cells, determination of individual prognoses may be more accurate and treatment may be facilitated due to a better understanding of this disease.

Acute lymphoblastic leukemia (ALL) and acute myeloblastic leukemia (AML) are heterogeneous groups of diseases characterized by malignant proliferation and accumulation of immature lymphoid and myeloid cells in the lymphoid organs, bone marrow, and blood. The neoplastic process that leads to leukemogenesis is characterized by cellular and molecular dysregulation leading to uncontrolled cell growth. Malignant cells often result from alterations to the genes controlling the cell cycle. These alterations may be due to genetic mutations or altered expression of affected genes.

Fas (CD95, APO-1) is a member of the tumor necrosis factor (TNF) superfamily. Ligation of the Fas receptor with Fas Ligand induces aggregation of Fas molecules, which creates an intracellular region designated a "death domain". This domain is essential for inducing caspase-dependent apoptosis in affected cells. ${ }^{4}$ Altered expression of Fas has been shown to render malignant cells less susceptible to killing by immune effector cells. Interestingly, certain chemotherapeutic agents have been shown to increase expression of Fas on malignant cells and cause Fas ligand-independent aggregation of Fas molecules, leading to apoptosis. 5,6 TNF-Related Apoptosis Inducing Ligand (TRAIL) is a molecule that has been shown to induce apoptosis primarily in malignant cells $^{7}$. TRAIL receptors include death receptors R1 and R2. Although chemotherapy sensitizes cancer cells to killing by TRAIL, it is not known whether apoptosis caused by anti- cancer drugs involves TRAIL receptor signaling. ${ }^{8}$ Given that both ligand-mediated and ligand-independent triggering of apoptosis through Fas and possibly TRAIL-R1 and -R2 are relevant to the success of chemotherapy, it is reasonable to hypothesize that alterations in the expression of molecules that regulate death receptor-mediated apoptosis may affect chemotherapy-resistance in malignant cells. Thus, it is important to examine the expression of regulatory molecules such as Fas-associated phosphatase (FAP)-1 and FLICE (caspase 8)-Inhibitory Protein (FLIP). To date, no studies have examined the expression of FAP-1 or FLIP in leukemias. The involvement of these anti-apoptotic molecules in leukemogenesis is uncertain.

The purpose of this study was to investigate the expression of Fas, TRAIL receptors, FAP-1, and FLIP by pediatric leukemia cells. For these preliminary analyses, leukemic cells were isolated from two pediatric patients suffering from ALL and AML who had not been previously treated for their malignancy. Expression was compared between these leukemic cells and Jurkat and CEM ALL cell lines, as well as normal circulating leukocytes isolated from non-leukemic pediatric patients, which were used as controls. This preliminary study will eventually enroll additional pediatric patients with leukemia.

\section{Methods}

Peripheral blood samples were obtained from pediatric patients treated at the Isaac Walton Killam (IWK) Health Centre. The IWK Research Ethics Board approved this study and its consent forms. Pediatric patients suffering from ALL and AML who were to undergo standard induction chemotherapy, but had not been previously treated for their malignancy, were selected for inclusion in the study. Peripheral blood samples from two patients with ALL were analyzed for this study. Blood was collected prior to induction chemotherapy and was processed immediately to prevent potential degradation or alteration of samples. Leukemic cells were isolated by standard procedures. Briefly, heparinized 
blood samples were separated by Ficoll-Hypaque density centrifugation at the time of collection. Purified leukocyte fractions were washed with phosphate-buffered saline (PBS). Normal circulating leukocytes isolated from non-leukemic pediatric patients were used as controls.

\section{RNA Isolation}

mRNA was isolated from $4 \times 10^{6}$ leukemic cells and control peripheral blood lymphocytes. Total RNA was isolated from T-cells using TRIzol (Gibco BRL) as per the manufacturer's instructions.

\section{Reverse transcription-polymerase chain reaction (RT- PCR) analysis}

Reverse transcription of mRNA was carried out in a $20 \mathrm{ml}$ volume containing $200 \mathrm{U}$ Moloney murine leukemia virus reverse transcriptase, $1 \mathrm{mg}$ random hexanucleotide primers, and $0.5 \mathrm{mM}$ dNTPs. The reaction mixtures were incubated at $37^{\circ} \mathrm{C}$ for 1 hour, and then at $95^{\circ} \mathrm{C}$ for 10 minutes. The final volume was adjusted to $200 \mathrm{ml}$ with pyrogen free water. cDNA in a total volume of $50 \mathrm{ml}$, along with $2.5 \mathrm{ml}$ Taq DNA polymerase, $0.2 \mathrm{mM}$ dNTPs, and $50 \mathrm{mM}$ of each primer were used for subsequent polymerase chain reaction (PCR). The PCR reaction mixture was covered with $100 \mathrm{ml}$ of mineral oil to prevent evaporation. The following primers were used for PCR (Table 1):

PCR amplification was achieved using the following protocols:

- b-actin: 26 cycles, denaturation $94^{\circ} \mathrm{C} 30$ s, annealing $57^{\circ} \mathrm{C}$ 30s, synthesis $72^{\circ} \mathrm{C} 60 \mathrm{~s}$

- Fas: 29 cycles, denaturation $94^{\circ} \mathrm{C} \mathrm{30s,} \mathrm{annealing} 57^{\circ} \mathrm{C} \mathrm{30s,}$ synthesis $72^{\circ} \mathrm{C} 60 \mathrm{~s}$

- TRAIL DR4: 29 cycles, denaturation $94^{\circ} \mathrm{C} 30$ s, annealing $57^{\circ} \mathrm{C} 30 \mathrm{~s}$, synthesis $72^{\circ} \mathrm{C} 90 \mathrm{~s}$

- TRAIL DR5: 29 cycles, denaturation $94^{\circ} \mathrm{C} 30$ s, annealing $57^{\circ} \mathrm{C} 30 \mathrm{~s}$, synthesis $72^{\circ} \mathrm{C} 90 \mathrm{~s}$

- FLIP: 32 cycles, denaturation $94^{\circ} \mathrm{C} 30$ s, annealing $57^{\circ} \mathrm{C}$ 30 s, synthesis $72^{\circ} \mathrm{C} 120 \mathrm{~s}$

- FAP-1: 34 cycles, denaturation $94^{\circ} \mathrm{C} 30$ s, annealing $57^{\circ} \mathrm{C}$ 30s, synthesis $72^{\circ} \mathrm{C} 90 \mathrm{~s}$

PCR products were visualized by electrophoresis on ethidium bromide stained $1.8 \%$ agarose gels. Relative abundance of PCR products was determined by densitometric analysis of gel scans. The number of PCR cycles selected for ampli- fication of cDNA was previously determined based on the generation of a PCR product during the exponential phase. For example, in order to determine the appropriate number of PCR cycles for determination of relative abundance of $\mathrm{mRNA}$ coding for b-actin, Fas, TRAIL DR4, TRAIL DR5, FLIP, and FAP-1, the amplification curve of each PCR product was determined by examining a range of cycles from 20 to 41 . The appropriate number of cycles was determined based on the number of cycles required to amplify a product during the exponential phase without reaching a plateau of amplification. RT-PCR performed under these conditions allows for semi-quantitative analysis of mRNA levels and the detection of twofold or greater differences in expression. ${ }^{9}$

\section{Results}

Cell viability is similar in malignant and normal lymphocytes

In order to assess whether there was a difference in the apoptotic-status of untreated normal and malignant cells, cell viability was measured by tryptan-blue exclusion. Jurkat ALL cells, CEM ALL cells, normal circulating lymphocytes, and circulating leukemic cells exhibited similar cell viability (Figure 1).

\section{Altered expression of regulatory genes FAP-1 and FLIP in $\mathbf{A L L}$ \\ Following the determination of the optimal number of PCR cycles (data not shown), mRNA expression of three apopto-}

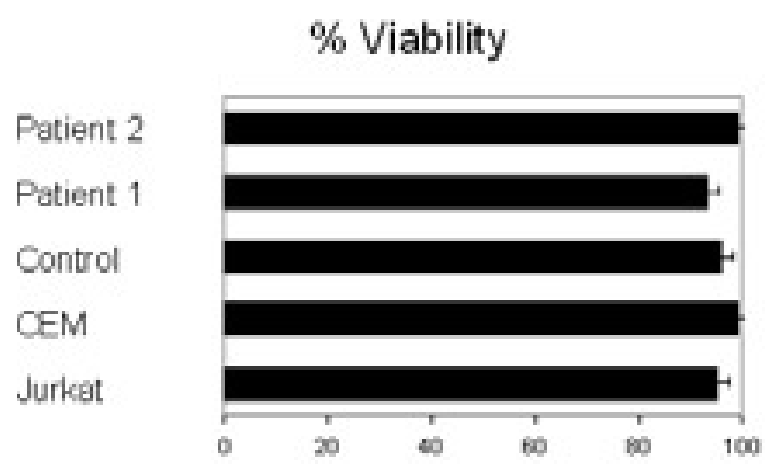

Figure 1: Viability of normal peripheral leukocytes and leukemic cell lines (CCRF-CEM and Jurkat), as assessed by trypan blue exclusion.

Table 1: The primers used for PCR

\begin{tabular}{|c|c|c|c|}
\hline Gene & Product Size & Primer Direction & Primer Sequence \\
\hline \multirow[t]{2}{*}{ b-actin } & 437 & Sense & 5'-TGGACATCCGCAAAGACCCGTACG-3' \\
\hline & & Antisense & 5'-TGTCACCTTCACCGTTCCAGTT-3' \\
\hline \multirow[t]{2}{*}{ Fas } & 369 & Sense & 5'-GAGACTCAGAACTTGGAAGGC-3' \\
\hline & & Antisense & 5'-CTTTGCACTTGGTGTTGCTGG-3' \\
\hline \multirow[t]{2}{*}{ TRAIL DR4 } & 502 & Sense & 5'-AGCAACGCAGACTCGCTGTCCA-3' \\
\hline & & Antisense & 5'-CCAAGGACACGGCAGAGCCTGT-3' \\
\hline \multirow[t]{2}{*}{ TRAIL DR5 } & 707 & Sense & 5'-CACCACGACCAGAAACACAG-3' \\
\hline & & Antisense & 5'-CGGTTTTGTTGACCCACTTT-3' \\
\hline \multirow[t]{2}{*}{ FLIP } & 945 & Sense & 5'-ATTGGTGAGGATTTGGATAAATCTG-3' \\
\hline & & Antisense & 5'-GTGGGCGTTTTCTTTCTTGTCTC-3' \\
\hline \multirow[t]{2}{*}{ FAP-1 } & 607 & Sense & 5'-GAATACGAGTGTCAGACATGG-3' \\
\hline & & Antisense & 5'-AGGTCTGCAGAGAAGCAAGAATAC-3' \\
\hline
\end{tabular}




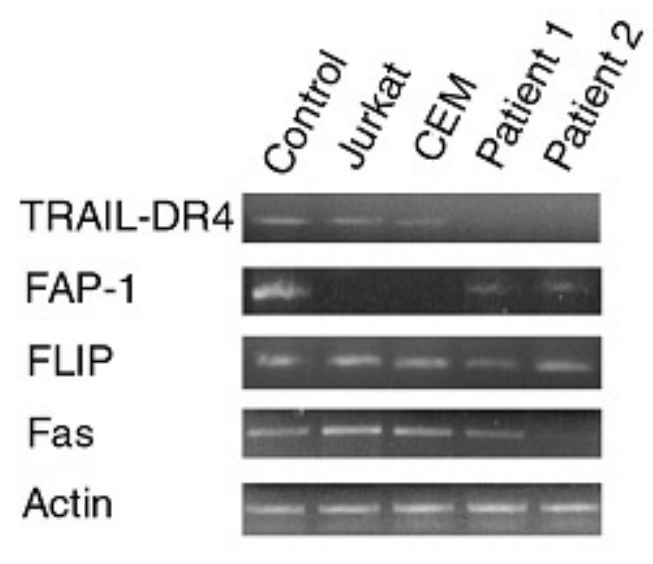

Figure 2: RT-PCR analysis of mRNA expression for FAP1, FLIP, Fas, and TRAIL-DR4 in normal peripheral leukocytes and leukemic cell lines (CCRF-CEM and Jurkat).

sis-inducing genes (Fas, DR4, and DR5) and two regulatory genes (FAP-1 and FLIP) that can modulate sensitivity to apoptotic cell death were investigated. As demonstrated in Figure 2, Fas mRNA expression was increased in ALL cell lines relative to normal circulating lymphocytes. Interestingly, Fas mRNA expression was decreased in patient samples relative to normal lymphocytes. TRAIL-DR4 mRNA expression was decreased in both ALL cell lines relative to normal lymphocytes and was undetectable in patient samples. FLIP mRNA expression was similar in normal circulating lymphocytes and in ALL cell lines, but was variably expressed in patient samples. FAP-1 mRNA expression was decreased in patient samples relative to normal circulating lymphocytes and was undetectable in ALL cell lines.

The primary objective of this study was to determine the relative mRNA expression of pro- and anti-apoptotic molecules in leukemic cells, as compared with normal lymphocytes. Results showed that differences observed in mRNA expression between normal circulating lymphocytes, ALL cell lines, and leukemic cells isolated from pediatric patients were not due to differences in the viability of these cells (Figure 1).

The data presented in this study indicate that expression levels of transcripts of Fas, TRAIL-DR4, and FAP-1 were altered in ALL cell lines and leukemic cells isolated from pediatric patients, relative to normal circulating lymphocytes. Since these molecules are involved in the regulation of the TRAIL- and Fas-mediated apoptotic pathways and certain chemotherapeutic agents induce apoptosis through Fas (and possibly TRAIL receptor) aggregation, a given leukemic cell clone may be more or less susceptible to chemotherapeutic measures based on its expression of these regulatory molecules. Decreased expression of pro-apoptotic receptors such as Fas and TRAIL-DR4 could conceivably contribute to the development of drug-resistance in malignant cells.

Fas expression was decreased in patient samples relative to normal circulating lymphocytes, but was increased in ALL cell lines. This finding suggests that the ALL cell lines may be more susceptible to Fas-mediated apoptosis than are circulat- ing leukemic cells in patients with leukemia. Considering that ALL cell lines are sensitive to cell death mediated by agonistic anti-Fas $\mathrm{mAb}$, it is possible that circulating leukemic cells isolated from patients may be less susceptible to the induction of apoptosis through Fas. This possibility will be examined in future studies.

TRAIL-DR4 mRNA expression was decreased in both ALL cell lines relative to normal lymphocytes and was undetectable in patient samples. These data suggest a potential mechanism of immune evasion by leukemic cells. Based on evidence that host immune surveillance is important for eliminating leukemic cells, the finding that leukemic cells express reduced levels of mRNA coding for pro-apoptotic TRAIL-receptors supports this possibility. ${ }^{10,11}$

FLIP mRNA expression was similar in all cells studied whereas FAP-1 expression was decreased or absent in patient samples and ALL cell lines, respectively (Figure 2). This finding suggests that FLIP may not be directly involved in the leukemogenesis. Decreased FAP-1 expression suggests that the role of FAP-1 in preventing apoptosis in leukemic cells is likely to be unimportant. A recent study reported that an increased FLIP:caspase- 8 ratio can decrease the susceptibility of cell lines to apoptosis, likely because FLIP modulates the activity of caspase- $8 .{ }^{12}$

The data presented in this study are based on preliminary research. With the provision that only two patients have been enrolled in this study to date, our preliminary data provide an indication of possible molecular differences in the regulation of apoptosis between non-malignant cells, leukemic cell lines, and circulating leukemic cells isolated from patient blood samples. The ultimate goal of this continuing study is to examine samples from 40 pediatric patients with ALL or AML, along with control leukocytes from 20 healthy individuals. It remains to be determined how the important proand anti-apoptotic molecules of interest are involved in the clinical treatment and prognosis of pediatric ALL and AML. This study seeks to explore the relevance of these molecules in the context of prognosis and clinical outcome. Increased mRNA expression of anti-apoptotic molecules or decreased expression of pro-apoptotic molecules could be risk factors for prognosis of ALL and AML.

\section{Conclusion}

Preliminary data shows that leukemic cells may express altered levels of mRNA coding for pro- and anti-apoptotic genes relative to normal lymphocytes. This research may reveal reasons for the variability of patient responses to induction chemotherapy. Such information is relevant when considering different treatment regimens because certain molecular characteristics may render leukemic cells resistant to particular therapies. As such, the results of further study in this area may help predict the likelihood of treatment success in individual patients based on the molecular characteristics of an individual's leukemic cells. The results of this study will also provide insight into the role of molecules involved in apoptosis in the process of leukemogenesis. 


\section{References}

1. Champlin R, Gale RP. Acute lymphoblastic leukemia: recent advances in biology and therapy. Blood 1989; 73: 2051-66.

2. Cortes JE, Kantarjian HM. Acute lymphoblastic leukemia: A comprehensive review with emphasis on biology and therapy. Cancer 1995; 76: 2393-417.

3. Hoelzer DF. Diagnosis and treatment of adult acute lymphocytic leukemia. In: Wiernik PH, Canellos GP, Dutcher JP, Kyle RA, eds. Neoplastic Diseases of the Blood. New York: Churchill Livingston, 1996: 295.

4. Timmer T, de Vries EG, de Jong S. Fas receptor-mediated apoptosis: A clinical application? J Pathol 2002; 196: 125-34.

5. Micheau, O, Solary E, Hammann A, Dimanche-Boitrel MT. Fas ligand-independent, FADD-mediated activation of the Fas death pathway by anticancer drugs. J Biol Chem 1999; 274: 7987-92.

6. Bush JA, Cheung KJ, and Li G. Curcumin induces apoptosis in human melanoma cells through a Fas receptor/Caspase-8 pathway independent of p53. Exp Cell Res 2001; 271: 305-14.

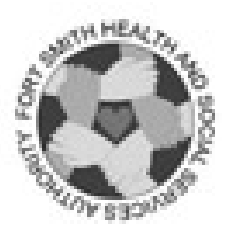

Fort Smith, the garden capital of the Northwest Territories is located on the banks of the Slave River just one mile from the Alberta/NWT border. This community provides a variety of social, recreational and cultural activities for it 3,000 residents, and is surrounded by a vast natural setting ideal for outdoor enthusiasts. Here you can spend the long summer days camping, hiking and kayaking along the many rivers, rapids and lakes in the area, or tending to your garden. Winter days can be filled with snowmobiling, cross-country skiing, snowshoeing and a whole lot more.

Our family physicians and health professionals provide health care services to patients in a twentytwo bed hospital, and the community.

Family Physicians

Physiotherapist

- Registered Nurses

Public Health Nurses

Licensed Practical Nurses

Contact Dana Rasiah, Chicf Exceutive Officer, Fort Smith Health \& Social Services Authority, P,O. Box 1080, Fort Smith, NT, X0-E 0P0, Phone: 867 872-6200, Fax: 867-872-6291, e-mail: dana_rasiah@gov.nt.ca
7. Gura T. How TRAIL kills cancer cells, but not normal cells. Science 1997; 277: 768.

8. Morgan M, Williams B, Blay J, Hoskin DW. Chemosensitization of T-47D breast carcinoma cells to TRAIL and Fas receptor-induced killing. Anticancer Res 2002; 22: 673-6.

9. Singer-Sam J, Robinson MO, Bellvé AR, Simon MI, Riggs AD. Measurement by quantitative PCR of changes in HPRT, PGK-1, PGK-2, APRT, MTase, and Zyf gene transcripts during mouse spermatogenesis. Nucleic Acids Res 1990; 18: 1255-1259.

10. Horowitz MM, Gale RP, Sondel PM, Goldman JM, Kersey J, Kolb HJ, Rimm AA, Ringden O, Rozman C, Speck B, Truitt RL, Zwaan FE, Bortin MM. Graft-versusleukemia reactions after bone marrow transplantation. Blood 1990; 75: 555-62.

11. Kersey JH. Fifty years of studies of the biology and therapy of childhood leukemia. Blood 1997; 90: 4243-51.

12. Tepper CG, Seldin MF. Modulation of caspase- 8 and FLICE-inhibitory protein expression as a potential mechanism of Epstein-Barr virus tumorigenesis in Burkitt's lymphoma. Blood 1999; 94: 1727-37.

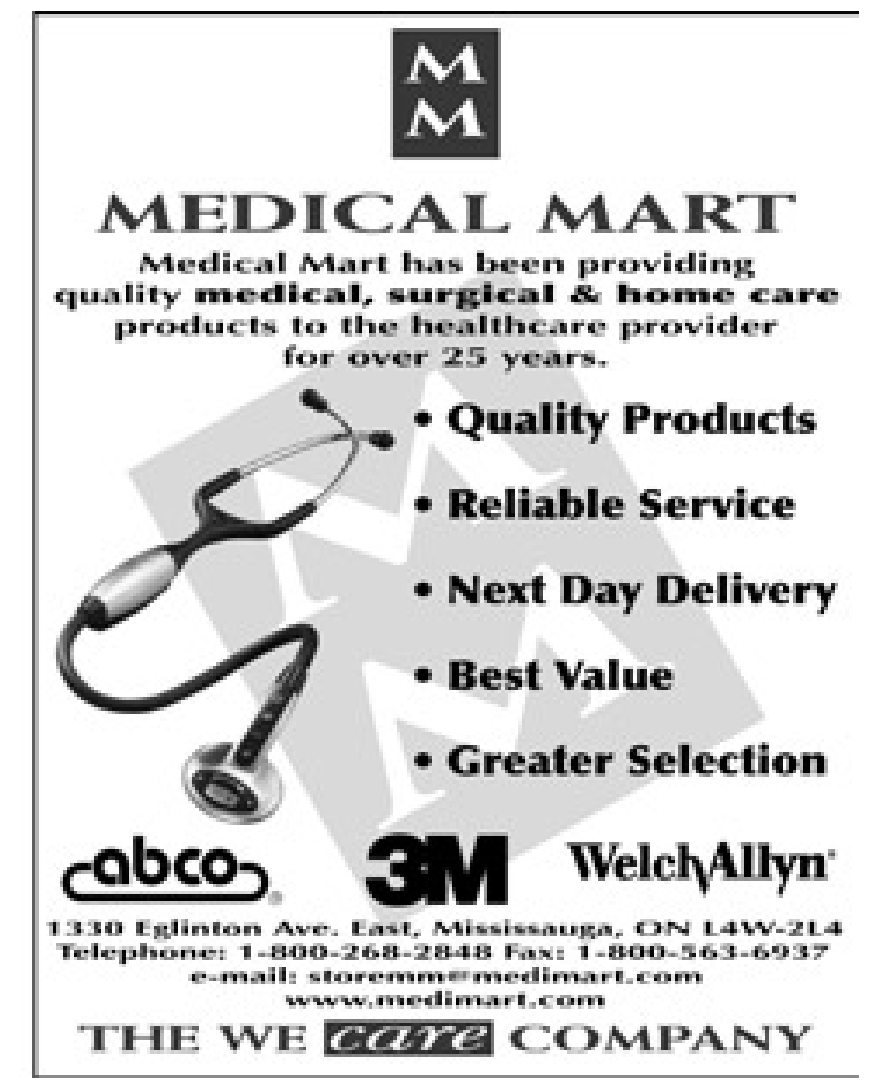

